\title{
Sotorasib: First Approval
}

\author{
Hannah A. Blair ${ }^{1}$ \\ Published online: 6 August 2021 \\ (c) Springer Nature, corrected publication 2021
}

\begin{abstract}
Sotorasib (LUMAKRAS ${ }^{\mathrm{TM}}$ ) is a RAS GTPase family inhibitor being developed by Amgen for the treatment of solid tumours with KRAS mutations, including non-small cell lung cancer (NSCLC) and colorectal cancer. In May 2021, sotorasib was granted accelerated approval by the US FDA for the treatment of adult patients with KRAS G12C-mutated locally advanced or metastatic NSCLC, as determined by an FDA-approved test, who have received at least one prior systemic therapy. This article summarizes the milestones in the development of sotorasib leading to this first approval for KRAS G12C-mutated NSCLC.
\end{abstract}

\section{Digital Features for this AdisInsight Report can be found at https:// doi.org/10.6084/m9.figshare.15078999}

\section{Sotorasib (LUMAKRAS ${ }^{\mathrm{m}}$ ): Key Points}

RAS GTPase family inhibitor being developed by Amgen for the treatment of KRAS G12C-mutated NSCLC

Received its first approval on 28 May 2021 in the USA Approved for the treatment of adult patients with KRAS G12C-mutated locally advanced or metastatic NSCLC, as determined by an FDA-approved test, who have received at least one prior systemic therapy

\section{Introduction}

KRAS, a GTPase and member of the RAS family of proteins, is the most frequently mutated oncogene in cancer [1]. The KRAS G12C mutation is present in approximately $13 \%$

This profile has been extracted and modified from the AdisInsight database. AdisInsight tracks drug development worldwide through the entire development process, from discovery, through preclinical and clinical studies to market launch and beyond.

Hannah A. Blair

dru@adis.com

1 Springer Nature, Mairangi Bay, Private Bag 65901, Auckland 0754, New Zealand of patients with lung cancer, $3 \%$ of patients with colorectal cancer and $2 \%$ of patients with other solid tumours [1, 2]. Unlike other mutant KRAS proteins, KRAS G12C has been shown to cycle between its active GTP-bound and inactive GDP-bound states within cancer cells [2], thereby providing a basis for the development of targeted therapies [2, 3].

Sotorasib (LUMAKRAS ${ }^{\mathrm{TM}}$ ) is a RAS GTPase family inhibitor being developed by Amgen for the treatment of solid tumours with KRAS G12C mutations. Sotorasib was given orphan drug designation by the US FDA in June 2019 for KRAS G12C-positive non-small cell lung cancer (NSCLC) and colorectal cancer [4]. The drug was granted breakthrough therapy designation for advanced or metastatic KRAS G12C-mutated NSCLC in December 2020 [5], and a priority review was granted in February 2021 [6]. On 28 May 2021, sotorasib received its first approval in the USA for the treatment of adult patients with KRAS G12C-mutated locally advanced or metastatic NSCLC, as determined by an FDA-approved test, who have received at least one prior systemic therapy [7, 8]. This indication was approved under accelerated approval based on overall response rate (ORR) and duration of response (DOR), and its continued approval may be contingent upon verification and description of clinical benefit in confirmatory trial(s) [7]. The recommended dosage of sotorasib is $960 \mathrm{mg}$ taken orally once daily (with or without food) until disease progression or unacceptable toxicity. Dosage modifications may be required because of adverse events (AEs). The recommended dose reduction levels are as follows: first reduction to $480 \mathrm{mg}$ once daily; second reduction to $240 \mathrm{mg}$ once daily. If patients are unable 


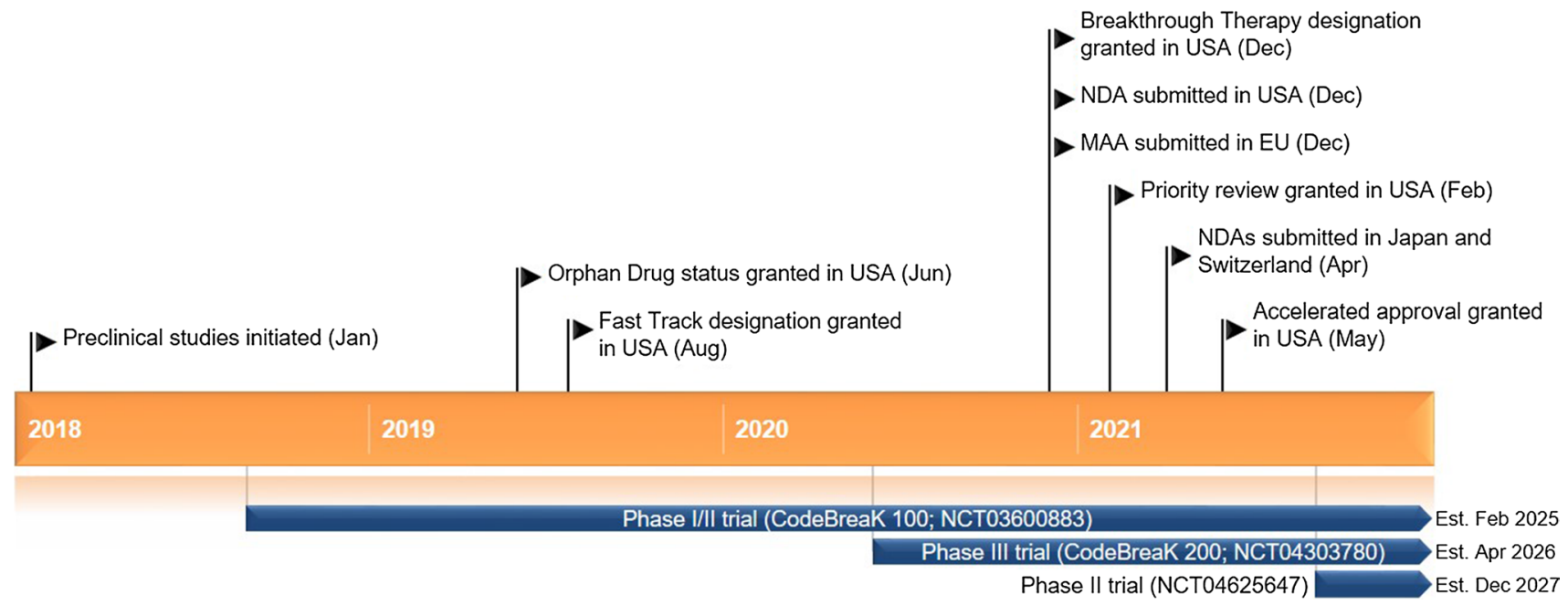

Key milestones in the development of sotorasib for the treatment of KRAS G12C-mutated non-small cell lung cancer. MAA Market Authorisation Application, NDA New Drug Application

to tolerate a dosage of $240 \mathrm{mg}$ once daily, treatment with sotorasib should be discontinued [7].

Phase I/II clinical trials of sotorasib in KRAS G12Cmutated colorectal cancer and other solid tumours are currently underway in multiple countries.

\subsection{Company Agreements}

In January 2014, Amgen and Carmot Therapeutics entered into a research, development and license agreement [9]. The agreement was extended in February 2016 [10] and December 2017 [11]. Under the terms of the agreement, Carmot Therapeutics will apply its proprietary lead-identification technology, Chemotype Evolution, to discover and develop drug leads for therapeutic targets selected by Amgen [9-11].

\subsection{Patent Information}

Amgen has patent protection for sotorasib in the USA, with an estimated expiration date of 2038 [12].

\section{Scientific Summary}

\subsection{Pharmacodynamics}

Sotorasib is a first-in-class KRAS G12C inhibitor. It binds covalently and irreversibly to the cysteine residue of the KRAS G12C mutant [7]. Consequently, the KRAS protein is locked in an inactive state and its downstream signalling effects are blocked, without affecting wild-type KRAS [7].

Sotorasib inhibited SOS1-catalyzed nucleotide exchange of recombinant mutant KRAS G12C/C118A in vitro [13].
Cysteine proteome analysis of cells treated with sotorasib demonstrated that only the G12C-containing peptide of KRAS was covalently modified. Sotorasib inhibited KRAS signalling (as measured by ERK phosphorylation) in all KRAS G12C mutant cell lines, but not in cell lines without the KRAS G12C mutation. Sotorasib also selectively impaired the viability of KRAS G12C mutant lines. Coadministration of sotorasib with inhibitors of other cellular signalling pathways afforded evidence for synergistic effects on cell viability [13].

In preclinical tumour models, sotorasib bound rapidly and irreversibly to KRAS G12C, thereby providing durable suppression of the mitogen-activated protein kinase (MAPK) signalling pathway [14]. Once daily administration of sotorasib led to tumour regression in mouse models of KRAS G12C cancer [14]. Sotorasib was also associated

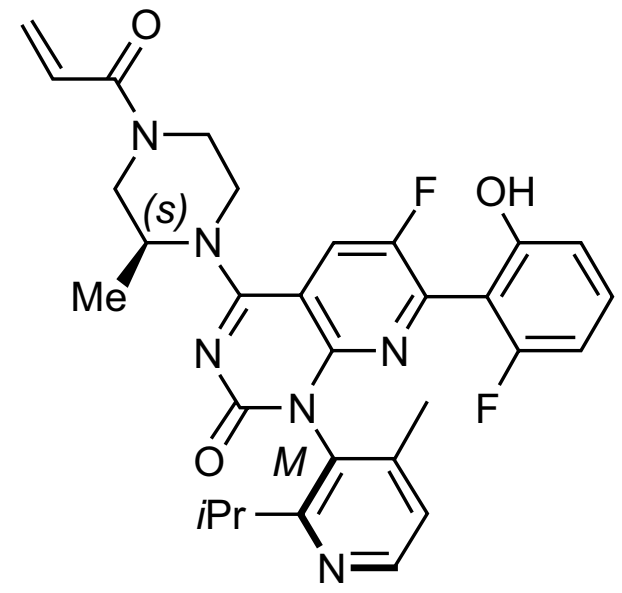

Chemical structure of sotorasib 
with prolonged survival and anti-tumour immunity in $K R A S$ G12C models [7].

The recommended dosage of sotorasib (i.e. $960 \mathrm{mg}$ once daily) was not associated with large mean increases (i.e. $>20 \mathrm{~ms}$ ) in the corrected QT interval [7].

\subsection{Pharmacokinetics}

Sotorasib demonstrates non-linear, time-dependent pharmacokinetics over a dose range of 180-960 mg once daily, with similar systemic exposure [i.e. area under the concentrationtime curve from zero to $24 \mathrm{~h}\left(\mathrm{AUC}_{0-24 \mathrm{~h}}\right)$ and maximum plasma concentration $\left(\mathrm{C}_{\max }\right)$ ] across doses at steady state [7]. Steady-state concentrations of sotorasib are achieved within 22 days, with no appreciable accumulation following repeated administration (mean accumulation ratio of 0.56). The median time to reach $\mathrm{C}_{\max }$ of sotorasib is $1 \mathrm{~h}$. Compared with fasting conditions, administration of a single dose of sotorasib $960 \mathrm{mg}$ with a high-fat, high-calorie meal increased sotorasib $\mathrm{AUC}_{0-24 \mathrm{~h}}$ by $25 \%$. Sotorasib is $89 \%$ bound to plasma proteins and has a mean volume of distribution at steady state of $211 \mathrm{~L} \mathrm{[7].}$

Sotorasib is largely metabolized by non-enzymatic conjugation and oxidative metabolism with CYP3As [7]. Following administration of a single radiolabeled dose of sotorasib, $74 \%$ of the dose was recovered in faeces (53\% as unchanged drug) and $6 \%$ in urine (1\% as unchanged drug). The mean apparent clearance of sotorasib at steady state is $26.2 \mathrm{~L} / \mathrm{h}$ and the mean terminal elimination half-life is $5 \mathrm{~h} \mathrm{[7].}$
Age (28-86 years), race (white, black or Asian), sex, body weight (36.8-157.9 kg), line of treatment, Eastern Cooperative Oncology Group performance status (ECOG PS; 0 or 1), mildly or moderately abnormal kidney function (estimated glomerular filtration rate $\geq 30 \mathrm{~mL} /$ $\min / 1.73 \mathrm{~m}^{2}$ ) and mild hepatic impairment [alanine aminotransferase (ALT) or aspartate aminotransferase (AST) $<2.5 \times$ upper limit of normal (ULN) or total bilirubin $<1.5 \times \mathrm{ULN}]$ did not affect the pharmacokinetics of sotorasib to a clinically significant extent [7]. The effect of severely abnormal kidney function or moderate to severe hepatic impairment on the pharmacokinetics of sotorasib is not known [7].

Coadministration of sotorasib with acid-reducing agents (e.g. omeprazole [15], famotidine [7]) or repeated doses of rifampicin (a strong CYP3A4 inducer) [16] decreased sotorasib $\mathrm{C}_{\max }$ and AUC. Coadministration of sotorasib with itraconazole [a combined strong CYP3A4 and P-glycoprotein (P-gp) inhibitor] [7], metformin (a MATE1/2K substrate) [17] or a single dose of rifampicin (an OATP1B1/1B3 inhibitor) $[7,16]$ did not affect the pharmacokinetics of sotorasib to a clinically significant extent. Sotorasib had no clinically relevant effect on the $\mathrm{C}_{\max }$ and $\mathrm{AUC}$ of metformin [17], but decreased the $\mathrm{C}_{\max }$ and AUC of the sensitive CYP3A4 substrate midazolam [7] and increased the $\mathrm{C}_{\max }$ and $\mathrm{AUC}$ of the $\mathrm{P}$-gp substrate digoxin [18]. In vitro, sotorasib may induce CYP2B6, CYP2C8 and CYP2C9 and may inhibit BCRP, but it does not inhibit CYP1A2, CYP2B6, CYP2C8, CYP2C9, CYP2C19 or CYP2D6 [7].

\section{Features and properties of sotorasib}

\begin{tabular}{|c|c|}
\hline Alternative names & AMG-510; LUMAKRAS ${ }^{\mathrm{TM}}$ \\
\hline Class & Anti-neoplastics, piperazines, pyridines, pyridones, pyrimidines, small molecules \\
\hline Mechanism of action & KRAS protein inhibitors \\
\hline Route of administration & Oral \\
\hline Pharmacodynamics & $\begin{array}{l}\text { Forms an irreversible, covalent bond with the cysteine residue of the } K R A S \mathrm{G} 12 \mathrm{C} \text { mutant, thereby locking } \\
\text { the protein in an inactive state and blocking its downstream signalling effects }\end{array}$ \\
\hline Pharmacokinetics & $\begin{array}{l}\text { Non-linear, time-dependent pharmacokinetics over dose range of } 180-960 \mathrm{mg} \text { once daily; median time to } \\
\mathrm{C}_{\max } 1 \mathrm{~h} \text {; mean Vd } 211 \mathrm{~L} \text {; mean apparent clearance } 26.2 \mathrm{~L} / \mathrm{h} \text {; mean } \mathrm{t}_{1 / 2} 5 \mathrm{~h}\end{array}$ \\
\hline \multicolumn{2}{|c|}{ Most frequent adverse events } \\
\hline Any grade & $\begin{array}{l}\text { Decreased lymphocytes, decreased haemoglobin, diarrhoea, increased AST, increased ALT, musculoskeletal } \\
\text { pain, decreased calcium, increased ALP, nausea, fatigue, hepatotoxicity, cough }\end{array}$ \\
\hline Grade 3 or 4 & Hepatotoxicity, increased ALT, increased AST, musculoskeletal pain, pneumonia, diarrhoea \\
\hline \multicolumn{2}{|l|}{ ATC codes } \\
\hline WHO ATC code & L01 (Anti-neoplastic agents) \\
\hline EphMRA ATC code & L1 (Anti-neoplastics) \\
\hline Chemical name & $\begin{array}{l}\text { 6-fluoro-7-(2-fluoro-6-hydroxyphenyl)-(1M)-1-[4-methyl-2-(propan-2-yl)pyridin-3-yl]-4-[(2S)-2-methyl-4- } \\
\text { (prop-2enoyl)piperazin-1-yl]pyrido[2,3-d]pyrimidin-2(1H)-one }\end{array}$ \\
\hline
\end{tabular}

$A L P$ alkaline phosphatase, $A L T$ alanine aminotransferase, $A S T$ aspartate aminotransferase, $C_{\max }$ maximum plasma concentration, $t_{1 / 2}$ terminal elimination half-life, $V d$ volume of distribution 


\subsection{Therapeutic Trials}

\subsubsection{Non-Small Cell Lung Cancer}

2.3.1.1 Phase II (Monotherapy) Sotorasib was associated with deep and durable responses in patients with locally advanced or metastatic KRAS G12C-mutated NSCLC in the registrational phase II portion of the ongoing, multicentre, phase I/II CodeBreaK 100 trial (NCT03600883) [19]. All patients had progressed on anti-programmed cell death protein 1 (PD-1) or anti-programmed death-ligand 1 (PD-L1) immunotherapy and/or platinum-based chemotherapy (and targeted therapy if EGFR, ALK and ROS1 alterations were identified) and had received $\leq 3$ prior lines of therapy. A total of 126 patients (median age 63.5 years) received oral sotorasib $960 \mathrm{mg}$ once daily until disease progression. The primary endpoint was confirmed objective response rate (ORR), assessed by blinded independent central review per RECIST 1.1 criteria [19].

At a median follow-up of 12.2 months (data cut-off 1 December 2020), the ORR was 37\% [19]. The disease control rate (DCR; defined as objective response or stable disease) was $81 \%$. The median time to response (TTR) was 1.4 months and the median DOR was 10.0 months. Median progression-free survival (PFS) was 6.8 months. At data cut-off, $43 \%$ of responders remained on treatment without disease progression [19]. Sotorasib was also associated with improvements in patient-reported outcomes, including global health status, quality of life, physical functioning and the severity of key lung cancer symptoms (e.g. cough, chest pain, dyspnoea) [20].
In exploratory analyses, the tumour response to sotorasib was seen across a range of biomarker subgroups, including patients with negative or low PD-L1 expression level and those with mutated STK11 [19]. The clinical benefit of sotorasib was also seen regardless of age ( $<65$ vs $\geq 65$ years), ECOG PS (0 vs 1$)$, metastatic disease (yes vs no), prior lines of therapy ( 1 vs $\geq 2$ ), prior anti-PD-1/PD-L1 therapy (yes vs no), TP53 co-mutation (wild-type vs mutant), STK11 comutation (wild-type vs mutant), KEAPI co-mutation (wildtype vs mutant) and tumour mutational burden (low vs high) [21].

2.3.1.2 Phase I (Dose Escalation and Expansion) Sotorasib demonstrated anti-cancer activity in patients with KRAS G12C-mutated NSCLC participating in the earlier phase I portion of CodeBreaK 100 [22]. Eligible patients (aged $\geq 18$ years) had histologically confirmed, locally advanced or metastatic NSCLC with the KRAS G12C mutation and had received previous platinum-based combination therapy, targeted therapies, or both. During the dose-escalation phase, patients received oral sotorasib $180,360,720$ or $960 \mathrm{mg}$ once daily in 21-day cycles until disease progression, unacceptable toxicity, withdrawal of consent or study end. A total of 59 patients with NSCLC were enrolled; of these, $90 \%$ had received previous anti-PD-1/PD-L1 therapies and $100 \%$ had received previous platinum-based chemotherapy [22].

At a median follow-up of 11.7 months (data cut-off 1 June 2020), the ORR was 32\% (all partial responses) and the DCR was $88 \%$ [22]. Responses were seen across all dose levels. Among those in the $960 \mathrm{mg} /$ day cohort $(n=34)$, the ORR was $35 \%$ and the DCR was $91 \%$. Tumour shrinkage of

Key clinical trials of sotorasib (Amgen)

\begin{tabular}{|c|c|c|c|c|c|}
\hline $\operatorname{Drug}(\mathrm{s})$ & Indication & Phase & Status & Location(s) & Identifier \\
\hline $\begin{array}{l}\text { Sotorasib, } \\
\text { docetaxel }\end{array}$ & NSCLC & III & Ongoing & Multinational & $\begin{array}{l}\text { CodeBreaK 200; NCT04303780; } \\
\text { EudraCT2019-003582-18 }\end{array}$ \\
\hline Sotorasib & NSCLC & $\mathrm{N} / \mathrm{A}^{\mathrm{a}}$ & Recruiting & Multinational & NCT04667234 \\
\hline Sotorasib & NSCLC & II & Not yet recruiting & Unknown & $\begin{array}{l}\text { NCT04625647; NCI-2020-08103; S1900E, } \\
\text { U10CA180888 }\end{array}$ \\
\hline $\begin{array}{l}\text { Sotorasib, } \\
\text { PD-1/PD-L1 } \\
\text { inhibitors }\end{array}$ & $\begin{array}{l}\text { NSCLC, colorectal } \\
\text { cancer, solid } \\
\text { tumours }\end{array}$ & $\mathrm{I} / \mathrm{II}$ & Recruiting & Multinational & $\begin{array}{l}\text { CodeBreaK 100; NCT03600883; } \\
\text { EudraCT2018-001400-11 }\end{array}$ \\
\hline $\begin{array}{l}\text { Sotorasib, } \\
\text { anti-cancer } \\
\text { therapies }\end{array}$ & $\begin{array}{l}\text { NSCLC, colorectal } \\
\text { cancer, solid } \\
\text { tumours }\end{array}$ & $\mathrm{Ib}$ & Recruiting & Japan, USA & CodeBreaK 101; NCT04185883 \\
\hline Sotorasib & Solid tumours & I & Recruiting & Hong Kong, Taiwan & CodeBreaK 105; NCT04380753 \\
\hline Sotorasib & $\begin{array}{l}\text { NSCLC, solid } \\
\text { tumours }\end{array}$ & I & Recruiting & USA & NCT04887064 \\
\hline
\end{tabular}

N/A not applicable, $N S C L C$ non-small cell lung cancer, $P D-1$ programmed cell death protein $1, P D$ - $L 1$ programmed death-ligand 1

${ }^{\text {a }}$ Expanded access protocol 
any magnitude was seen in $71 \%$ of patients after 6 weeks. The median TTR was 1.4 months and the median DOR was 10.9 months. Median PFS was 6.3 months [22].

\subsubsection{Colorectal Cancer}

Sotorasib demonstrated clinical activity in patients with colorectal cancer participating in the phase I portion of CodeBreaK $100(n=42)$ [22]. All patients had received at least two previous lines of systemic therapy for metastatic colorectal cancer. At a median follow-up of 12.8 months (data cut-off 1 June 2020), 7\% of patients had an ORR (all partial responses) and $74 \%$ of patients had disease control. The median duration of stable disease was 5.4 months. In the cohort receiving sotorasib $960 \mathrm{mg} /$ day $(n=25)$, the ORR was $12 \%$ and the DCR was $80 \%$. Median PFS was 4.0 months [22].

\subsubsection{Other Solid Tumours}

In patients with other solid tumours participating in the phase I portion of CodeBreaK $100(n=28)$, sotorasib was associated with an ORR of $14 \%$ and a DCR of $75 \%$ [22]. Partial responses were seen in patients with pancreatic cancer, endometrial cancer, appendiceal cancer and melanoma (all $n=1$ ). Five patients remained on treatment at the time of data cut-off [22].

\subsection{Adverse Events}

Sotorasib $960 \mathrm{mg}$ once daily had a manageable tolerability profile in the subset of 204 patients with KRAS G12Cmutated NSCLC enrolled in CodeBreaK 100 [7]. The most common (incidence $\geq 20 \%$ ) AEs in patients receiving sotorasib were diarrhoea (42\%), musculoskeletal pain (35\%), nausea $(26 \%)$, fatigue (26\%), hepatotoxicity (25\%) and cough (20\%). The most common (incidence $\geq 30 \%$ ) laboratory abnormalities were decreased lymphocytes (48\%), decreased haemoglobin (43\%), increased AST (39\%), increased ALT $(38 \%)$, decreased calcium (35\%) and increased alkaline phosphatase (33\%). The most common (incidence $\geq 5 \%$ ) grade 3 or $4 \mathrm{AEs}$, including laboratory abnormalities, were hepatotoxicity (12\%), increased ALT (11\%), increased AST (9\%), musculoskeletal pain (8\%), pneumonia (7\%) and diarrhoea (5\%) [7].

Serious AEs occurred in $50 \%$ of patients receiving sotorasib, with pneumonia (8\%), hepatotoxicity (3\%) and diarrhoea (2\%) reported most frequently (incidence $\geq 2 \%$ ) [7]. Fatal AEs occurred in $3 \%$ of sotorasib recipients (respiratory failure, pneumonitis, cardiac arrest, cardiac failure, gastric ulcer and pneumonia). AEs led to permanent discontinuation of sotorasib in $9 \%$ of patients, with hepatotoxicity (5\%) being the most common (incidence $\geq 2 \%$ ) reason for discontinuing treatment. Sotorasib dose reductions because of AEs were required in 5\% of patients and dosage interruptions because of AEs in $34 \%$ of patients. Dose reductions were most commonly (incidence $>2 \%$ ) for increased ALT (3\%) and increased AST (3\%), and dosage interruptions were required most frequently (incidence $\geq 2 \%$ ) for hepatotoxicity ( $11 \%$ ), diarrhoea (8\%), musculoskeletal pain (4\%), nausea (3\%) and pneumonia (3\%) [7]. Most patients reported that they were 'not at all' (54-79\%) or 'a little bit' $(8-33 \%)$ bothered by sotorasib side effects on the GP5 item of the Functional Assessment of Cancer Therapy-General questionnaire [20].

The US prescribing information contains a warning stating that sotorasib may cause hepatotoxicity and potentially fatal interstitial lung disease (ILD)/pneumonitis [7]. In the pooled safety population of 357 patients with NSCLC and other solid tumours with KRAS G12C mutation enrolled in CodeBreaK 100, the incidence of grade 3 hepatotoxicity was $1.4 \%$. Grade 3 and 4 ALT/AST elevations occurred in $6 \%$ and $0.6 \%$ of patients. The median time to first onset of increased ALT/AST was 9 weeks. Grade 3 or 4 ILD/pneumonitis occurred in $0.8 \%$ of patients; one case was fatal. The median time to first onset of ILD/pneumonitis was 2 weeks [7].

\subsection{Companion Diagnostic}

The US FDA has approved Guardant $360^{\circledR} \mathrm{CDx}$, a bloodbased liquid biopsy companion diagnostic developed by Guardant Health, for the identification of the KRAS G12C mutation in patients with NSCLC [23]. The therascreen ${ }^{\circledR}$ KRAS RGQ PCR Kit developed by QIAGEN has also been approved by the US FDA as a tissue-based companion diagnostic to identify the KRAS G12C mutation in NSCLC tumours [24].

\subsection{Ongoing Clinical Trials}

In addition to the ongoing phase I/II CodeBreaK 100 trial described above (NCT03600883), the randomized, open-label, multicentre, phase III CodeBreaK 200 trial (NCT04303780) is underway. CodeBreaK 200 will evaluate the efficacy and tolerability of sotorasib versus docetaxel in patients with previously treated advanced NSCLC harbouring the KRAS G12C mutation [25]. A phase II lung cancer master protocol (Lung MAP) trial (NCT04625647) plans to evaluate the efficacy and tolerability of sotorasib in patients with stage IV or recurrent KRAS G12C-mutated non-squamous NSCLC.

The open-label, multicentre, phase Ib CodeBreaK 101 trial (NCT04185883) is currently recruiting patients. The trial will evaluate the safety, tolerability and efficacy of sotorasib alone and in combination with other anti-cancer 
therapies in patients with advanced KRAS G12C-mutated solid tumours, including NSCLC and colorectal cancer [26]. Patients are also being recruited in CodeBreaK 105 (NCT04380753), an open-label, multicentre, phase I ethnic sensitivity study which will evaluate the safety, tolerability, pharmacokinetics and efficacy of sotorasib in patients of Chinese descent with KRAS G12C-mutated advanced/ metastatic solid tumours.

Furthermore, an expanded access programme (NCT04667234) will provide treatment access to sotorasib and assess its safety in patients with previously treated locally advanced/unresectable/metastatic KRAS G12Cmutated NSCLC who are ineligible to participate in any ongoing sotorasib clinical trial.

\section{Current Status}

Sotorasib received its first approval on 28 May 2021 in the USA for the treatment of adult patients with KRAS G12Cmutated locally advanced or metastatic NSCLC, as determined by an FDA-approved test, who have received at least one prior systemic therapy [8].

Supplementary Information The online version contains supplementary material available at https://doi.org/10.1007/s40265-021-01574-2.

\section{Declarations}

Funding The preparation of this review was not supported by any external funding.

\begin{abstract}
Authorship and Conflict of interest During the peer review process, the manufacturer of the agent under review was offered an opportunity to comment on the article. Changes resulting from any comments received were made by the authors on the basis of scientific completeness and accuracy. Hannah Blair is a salaried employee of Adis International Ltd/Springer Nature, and declares no relevant conflicts of interest. All authors contributed to the review and are responsible for the article content.
\end{abstract}

Ethics approval, Consent to participate, Consent to publish, Availability of data and material, Code availability Not applicable.

Open Access This article is licensed under a Creative Commons Attribution-NonCommercial 4.0 International License, which permits any non-commercial use, sharing, adaptation, distribution and reproduction in any medium or format, as long as you give appropriate credit to the original author(s) and the source, provide a link to the Creative Commons licence, and indicate if changes were made. The images or other third party material in this article are included in the article's Creative Commons licence, unless indicated otherwise in a credit line to the material. If material is not included in the article's Creative Commons licence and your intended use is not permitted by statutory regulation or exceeds the permitted use, you will need to obtain permission directly from the copyright holder. To view a copy of this licence, visit http://creativecommons.org/licenses/by-nc/4.0/.

\section{References}

1. Canon J, Rex K, Saiki AY, et al. The clinical KRAS(G12C) inhibitor AMG 510 drives anti-tumour immunity. Nature. 2019;575(7781):217-23.

2. Kim D, Xue JY, Lito P. Targeting KRAS(G12C): from inhibitory mechanism to modulation of antitumor effects in patients. Cell. 2020;183(4):850-9.

3. Singh RR, O'Reilly EM. New treatment strategies for metastatic pancreatic ductal adenocarcinoma. Drugs. 2020;80(7):647-69.

4. Amgen. Amgen announces first clinical data evaluating novel investigational KRASG12C inhibitor AMG 510 at ASCO 2019 [media release]. 3 Jun 2019. https://www.amgen.com/ newsroom/press-releases/2019/06/amgen-announces-firstclinical-data-evaluating-novel-investigational-krasg 12c-inhib itor-amg-510-at-asco-2019.

5. Amgen. Amgen's sotorasib granted breakthrough therapy designation for advanced or metastatic non-small cell lung cancer patients with KRAS G12C mutation [media release]. 8 Dec 2020. https:// www.amgen.com/newsroom/press-releases/2020/12/amgens-sotor asib-granted-breakthrough-therapy-designation-for-advanced-ormetastatic-nonsmall-cell-lung-cancer-patients-with-kras-g12cmutation.

6. Amgen. FDA grants sotorasib priority review designation for the treatment of patients with KRAS G12C-mutated locally advanced or metastatic non-small cell lung cancer [media release]. $16 \mathrm{Feb}$ 2021. https://www.amgen.com/newsroom/press-releases/2021/ 02/fda-grants-sotorasib-priority-review-designation-for-the-treat ment-of-patients-with-kras-g12c-mutated-locally-advanced-ormetastatic-non-small-cell-lung-cancer.

7. Amgen Inc. LUMAKRASTM (sotorasib) tablets, for oral use: US prescribing information. 2021. https://www.accessdata.fda.gov/ drugsatfda_docs/label/2021/214665s000lbl.pdf. Accessed 5 July 2021.

8. US Food \& Drug Administration. FDA grants accelerated approval to sotorasib for KRAS G12C mutated NSCLC [media release]. 28 May 2021. https://www.fda.gov/drugs/drug-appro vals-and-databases/fda-grants-accelerated-approval-sotorasibkras-g12c-mutated-nsclc.

9. Carmot Therapeutics. Carmot Therapeutics enters into drug discovery collaboration with Amgen [media release]. 14 Jan 2014. https://www.businesswire.com/news/home/20140114005428/en/ Carmot-Therapeutics-Enters-into-Drug-Discovery-Collaborat ion-with-Amgen.

10. Carmot Therapeutics. Carmot Therapeutics extends drug discovery collaboration with Amgen [media release]. 16 Feb 2016. https://carmot-therapeutics.us/carmot-therapeutics-extends-drugdiscovery-collaboration-with-amgen/.

11. Carmot Therapeutics. Carmot enters a multi-year drug discovery collaboration with Amgen [media release]. 6 Dec 2017. https:// carmot-therapeutics.us/carmot-enters-a-multi-year-drug-disco very-collaboration-with-amgen-collaboration-aims-to-identifynovel-molecules-intended-for-parkinsons-disease/.

12. Amgen. Amgen letter to shareholders. 2020. https://investors. amgen.com/static-files/c1b90d85-8945-4d11-8d1c-5ddc343fb6 99. Accessed 5 July 2021.

13. Saiki AY, Gaida K, Rex K, et al. Discovery and in vitro characterization of AMG 510 - a potent and selective covalent smallmolecule inhibitor of $\mathrm{KRAS}^{\mathrm{G} 12 \mathrm{C}}$ [abstract no. 4484]. Cancer Res. 2019;79(13 Suppl.).

14. Lanman BA, Chen JJ, Liu L, et al. Discovery of AMG 510, a first-in-human covalent inhibitor of KRAS ${ }^{\mathrm{G} 12 \mathrm{C}}$ for the treatment of solid tumors [abstract no. 4455]. Cancer Res. 2019;79(13 Suppl.).

15. Cardona P, Simiens M, Purkis J, et al. An open-label study to evaluate the effect of omeprazole on the pharmacokinetics of 
sotorasib (AMG 510) in healthy subjects [abstract no. PII-009]. Clin Pharmacol Ther. 2021;109(Suppl. 1):S31.

16. Cardona P, Spring M, Purkis J, et al. An open-label study to evaluate the effect of single and multiple doses of rifampin on the pharmacokinetics of sotorasib (AMG 510) in healthy subjects [abstract no. PII-010]. Clin Pharmacol Ther. 2021;109(Suppl. 1):S31.

17. Vuu I, Simiens M, Purkis J, et al. A phase I, open-label study to evaluate drug-drug interactions between metformin and sotorasib (AMG 510) in healthy subjects [abstract no. PIII-018]. Clin Pharmacol Ther. 2021;109(Suppl. 1):S50.

18. Cardona P, Spring M, Purkis J, et al. An open-label study to evaluate the effect of sotorasib (AMG 510) on digoxin pharmacokinetics in healthy subjects [abstract no. PII-011]. Clin Pharmacol Ther. 2021;109(Suppl. 1):S31.

19. Li B, Skoulidis F, Falchook G, et al. Registrational phase 2 trial of sotorasib in KRAS p.G12C mutant NSCLC: first disclosure of the Codebreak 100 primary analysis [abstract no. PS01.07 + oral presentation]. J Thorac Oncol. 2021;16(3 Suppl.):S61.

20. Spira A, Wilson FH, Shapiro G, et al. Patient-reported outcomes (PRO) from the phase 2 CodeBreaK 100 trial evaluating sotorasib in KRAS p.G12C mutated non-small cell lung cancer (NSCLC) [abstract no. 9057]. J Clin Oncol. 2021;39(15 Suppl.):474s.

21. Skoulidis F, Li BT, Govindan R, et al. Overall survival and exploratory subgroup analyses from the phase 2 CodeBreaK 100 trial evaluating sotorasib in pretreated KRAS p.G12C mutated non-small cell lung cancer [abstract no. 9003]. J Clin Oncol. 2021;39(15 Suppl.):460s.

22. Hong DS, Fakih MG, Strickler JH, et al. KRAS ${ }^{\mathrm{G} 12 \mathrm{C}}$ inhibition with sotorasib in advanced solid tumors. N Engl J Med. 2020;383(13):1207-17.
23. Guardant Health. Guardant $360^{\circledR}$ CDx receives FDA approval as first and only liquid biopsy companion diagnostic for Amgen's

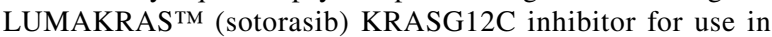
advanced non-small cell lung cancer [media release]. 28 May 2021. https://investors.guardanthealth.com/press-releases/ press-releases/2021/Guardant360-CDx-Receives-FDA-Appro val-as-First-and-Only-Liquid-Biopsy-Companion-Diagnosticfor-Amgens-LUMAKRAS-sotorasib-KRASG12C-Inhibitor-forUse-in-Advanced-Non-Small-Cell-Lung-Cancer/default.aspx.

24. QIAGEN. QIAGEN launches first FDA-approved tissue companion diagnostic to identify the KRAS G12C mutation in NSCLC tumours and expand precision medicine options in lung cancer [media release]. 28 May 2021. https://corporate.qiagen.com/newsr oom/press-releases/press-release-details/2021/QIAGEN-Launc hes-First-FDA-approved-Tissue-Companion-Diagnostic-to-Ident ify-the-KRAS-G12C-Mutation-in-NSCLC-Tumours-and-ExpandPrecision-Medicine-Options-in-Lung-Cancer/default.aspx.

25. Reck M, Spira A, Besse B, et al. CodeBreak 200: a phase III multicenter study of sotorasib (AMG 510), a KRAS(G12C) inhibitor, versus docetaxel in patients with previously treated advanced non-small cell lung cancer (NSCLC) harboring KRAS p.G12C mutation [abstract no. 1416TiP]. Ann Oncol. 2020;31(Suppl. 4):S894-5.

26. Hong DS, Strickler J, Fakih M, et al. Trial in progress: a phase $1 \mathrm{~b}$ study of sotorasib, a specific and irreversible KRAS ${ }^{\mathrm{G} 12 \mathrm{C}}$ inhibitor, as monotherapy in non-small cell lung cancer (NSCLC) with brain metastasis and in combination with other anticancer therapies in advanced solid tumors (CodeBreaK 101) [abstract no. TPS2669]. J Clin Oncol. 2021;39(15 Suppl.):145s. 